\title{
Coupling Modeling for Functional Surface of Electronic Equipment
}

\author{
Baoyan DUAN ${ }^{1} \cdot$ Congsi WANG $^{1} \cdot$ Wei WANG ${ }^{1}$
}

Published online: 11 April 2017

(C) The Author(s) 2017. This article is an open access publication

High performance electromechanical equipment is widely used in various fields, such as national defense, industry and so on [1]. In addition, the technical level of high performance electromechanical equipment is the embodiment of the national level of science and technology. There are two types of complex electromechanical equipment. One of them belongs to traditional machinery, in which the electronic information technology is used to enhance the mechanical performance by making the system more sophisticated, more efficient and more intelligent, such as numerical control machine. The other belongs to electronic equipment, in which the mechanics is used to enhance its electrical performance, such as microwave antenna, radome and so on.

At present, the electronic equipment as the representation of the second type of electromechanical equipment is utilized in various areas on land, in the sea, in the air and in outer space. There is a class of geometrical shape called functional surface in electronic equipment. It is a complex surface that realizes the specific physical properties. Different from the surface of traditional electromechanical equipment, such as turbine blades, aircraft shape, etc., functional surface is designed to achieve electricity, magnetic, light, heat and other functional characteristics of nontraditional mechanical disciplines.

The typical functional surfaces include reflector antenna, active phased array antenna, radome and so on. There is a nonlinear relationship (Fig. 1) between mechanical factors and electrical performances [2], which

Wei WANG

wwang@xidian.edu.cn

1 Key Laboratory of Electronic Equipment Structure Design of Ministry of Education, Xidian University, Xi'an 710071, China belongs to the electromechanical coupling issue of functional surfaces. Further study on this issue is helpful to improve the design level of electronic equipment.

After years of research, key laboratory of electronic equipment structure design of ministry of education has achieved some outstanding achievements on electromechanical coupling issue of functional surfaces, some of which have been applied in practical engineering. In the following, the electromechanical coupling issues and the correspond models for reflector antennas, active phased array antennas and radomes are introduced respectively.

\section{A. Reflector Antenna}

Reflector antennas are widely used in radar, communication, remote sensing, radio astronomy and other fields. However, with the development of modern reflector antennas towards large aperture and high frequency, it is more difficult to ensure their surface accuracy. Therefore, lots of work has been done to ensure their electrical performances from the coupling relationship between structural error and electrical performance [3]. But how to ensure the electrical performance of the antenna from the aspect of electromechanical coupling is the content that we want to present in the following.

The main factors related to the electrical properties of reflector antennas are panel deformation, feed position and attitude, etc. In practice, external loads such as gravity, wind and temperature will lead to the reflector panel's deformation, feed position offset and attitude deflection, which affect the electrical performance.

In order to express the influence of these mechanical factors on electrical performance, the electromechanical coupling model is established as follows [4]: 


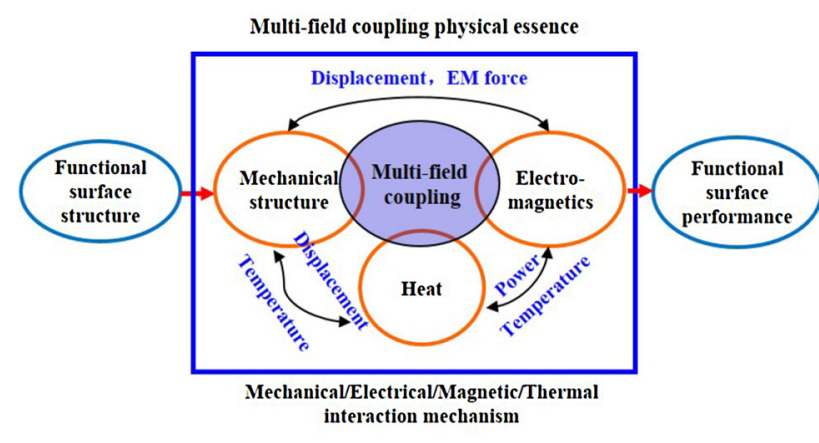

Fig. 1 Coupling modeling and influence mechanism of functional surface

$$
\begin{aligned}
E(\theta, \phi)= & \iint_{A} \frac{f_{0}\left(\xi-\Delta \xi(\boldsymbol{\delta}(\boldsymbol{\beta})), \phi^{\prime}-\Delta \phi^{\prime}(\boldsymbol{\delta}(\boldsymbol{\beta}))\right)}{\left|\boldsymbol{r}_{0}\right|} \\
& \exp \left[\mathrm{j} k \rho^{\prime} \sin \theta \cos \left(\phi-\phi^{\prime}\right)\right] \cdot \exp \left\{\mathrm { j } \left[\varphi_{f}(\boldsymbol{\delta}(\boldsymbol{\beta}))\right.\right. \\
& \left.\left.+\varphi_{s}(\boldsymbol{\delta}(\boldsymbol{\beta}))+\varphi_{r}(\gamma)\right]\right\} \rho^{\prime} \mathrm{d} \rho^{\prime} \mathrm{d} \phi^{\prime},
\end{aligned}
$$

where $k$ is the air propagation constant; $f_{0}(\xi-$ $\left.\Delta \xi(\boldsymbol{\delta}(\boldsymbol{\beta})), \phi^{\prime}-\Delta \phi^{\prime}(\boldsymbol{\delta}(\boldsymbol{\beta}))\right)$ is feed pointing error on aperture field amplitude; $\varphi_{f}(\boldsymbol{\delta}(\boldsymbol{\beta}))$ is feed location error on aperture field amplitude; $\varphi_{s}(\boldsymbol{\delta}(\boldsymbol{\beta}))$ is panel distortion on aperture field phase; $\varphi_{r}(\gamma)$ is panel machining accuracy on aperture field phase; $\boldsymbol{\beta}$ is structural design variables including size, shape, topology, type etc., and $\gamma$ is manufacturing process factor.

The electromechanical coupling model has been applied to a $66 \mathrm{~m}$ antenna structural design, which achieves the optimal stiffness distribution of the back-up structure and the center body, improves the structural symmetry and reduces the system failure risk. Ultimately, the gain of antenna increases by $0.4 \mathrm{~dB}$, the sidelobe level decreases by $1.2 \mathrm{~dB}$, and the weight decreases by $8 \%$.

\section{B. Active Phased Array Antenna}

The active phased array antenna has its own advantages, such as interference rejection and beam steering without physically move. In practice, the antenna structure also deforms under external loads. Accordingly, the surface error can dramatically degrade the radiation performance of active phased array antennas. There are many techniques for analyzing the radiation of array antennas considering mechanical errors without considering the mutual coupling effect. However, the mutual coupling is an indispensable factor for all practical considerations [5]. Because the mechanical distortion not only affects phase parameters and patterns of radiating elements, but also changes mutual coupling effect [6]. The electromechanical coupling analysis model is given as follows:

$$
\begin{aligned}
E(\theta, \phi)= & \sum_{n=1}^{N}\left[\sum_{m=1}^{N} C_{n m}(\boldsymbol{\delta}(\boldsymbol{\beta}, \boldsymbol{T}), \gamma) I_{m} \exp \left(\mathrm{j} \varphi_{m}\right)\right] \\
& \cdot f_{n}(\theta, \phi, \boldsymbol{\delta}(\boldsymbol{\beta}, \boldsymbol{T}), \gamma) \exp \left[\mathrm{j} k \boldsymbol{r}_{n}(\boldsymbol{\delta}(\boldsymbol{\beta}, \boldsymbol{T}), \gamma) \cdot \hat{\boldsymbol{r}}_{0}\right],
\end{aligned}
$$

where $N$ is the total number of radiating elements, $C_{n m}$ is mutual coupling parameter, $I_{m}, \varphi_{m}, f_{n}$, and $\boldsymbol{r}_{n}$ are excitation magnitude, excitation phase, pattern and position vector of the radiating element, respectively, and $\hat{\boldsymbol{r}}_{0}$ is spatial unit vector. The remaining symbols are the same as that of formula (1). The radiation performance of deformed array antenna can be analyzed using the analysis model.

\section{Radome}

The radome is a cover made of natural or manmade dielectric material, or a special shaped electromagnetic window made of a truss-supported dielectric housing. It provides a suitable interface for maintaining the structure, temperature and aerodynamic characteristics [7]. In the manufacturing process, complex molding is needed to obtain a specific shape. This results in inconsistent material parameters of the shell and affect the electrical properties.

After transmitting through the radome, the amplitude and phase distribution in antenna aperture will be changed. The influence of dielectric shell on amplitude and phase distribution can be characterized by transmission coefficients. The passed aperture field can be obtained, from which the far field can also be obtained [7].With the phase distribution of aperture field considered, the far field of radome-enclosed antenna can be written as

$$
\begin{aligned}
E_{t}(\theta, \phi)= & \iint_{A^{\prime}} f_{0}(x, y) \exp [\mathrm{j} k \sin \theta(x \cos \phi+y \sin \phi)] \\
& \cdot \exp \left[\mathrm{j} \varphi_{f}(x, y)\right] T(n+\Delta \boldsymbol{n}, \varepsilon+\Delta \varepsilon, d+\Delta d) \mathrm{d} x \mathrm{~d} y,
\end{aligned}
$$

where $E_{t}(\theta, \phi)$ is field value in the far-field, $A$ denotes antenna aperture while $A^{\prime}$ denotes antenna aperture after transmitting through radome, $f_{0}(x, y)$ and $\varphi_{f}(x, y)$ are amplitude and phase distribution, $T(n+\Delta \boldsymbol{n}, \varepsilon+\Delta \varepsilon, d+\Delta d) \quad$ is transmission coefficient of radome considering deformation and material errors, $\Delta \boldsymbol{n}$ is variation of normal vector, $\Delta \varepsilon$ is permittivity variation of radome material, and $\Delta d$ is thickness variation of radome wall.

Equation (3) can be applied to analyze the effect of material properties errors of streamlined airborne radome on the electrical performance. Besides, the thickness profile of the radome can be designed with optimization models [8], which has reference value for practical engineering.

With the development of electronic equipment toward high accuracy, high density, high frequency and atrocious 
service environment, the electromechanical coupling issues of functional surface will be more prominent, such as the passive intermodulation and the heat transfer problem, etc. Therefore, it is necessary to study the electromechanical coupling issues of electronic equipment in depth.

Open Access This article is distributed under the terms of the Creative Commons Attribution 4.0 International License (http://crea tivecommons.org/licenses/by/4.0/), which permits unrestricted use, distribution, and reproduction in any medium, provided you give appropriate credit to the original author(s) and the source, provide a link to the Creative Commons license, and indicate if changes were made.

\section{References}

1. HAUPT R L, RAHMAT-SAMII Y. Antenna array developments: A perspective on the past, present and future[J]. IEEE Antennas and Propagation Magazine, 2015, 57(1): 86-96.

2. DUAN B Y. Review of electromechanical coupling of electronic equipment[J]. SCIENCE CHINA Information Sciences, 2015, 45(3): 299-312. (in Chinese).

3. RAHMAT-SAMII Y, HAUPT R L. Reflector antenna developments: a perspective on the past, present and future[J]. IEEE Antennas and Propagation Magazine, 2015, 57(2): 85-95.

4. DUAN B Y, WANG C S. Reflector Antenna Distortion Analysis Using MEFCM[J]. IEEE Transactions on Antennas \& Propagation, 2009, 57(10): 3409-3413.

5. CRAEVE C, GONZALEZ-OVEJERO D. A review on array mutual coupling analysis[J]. Radio Science, 2011, 46(2): 1-25.

6. WANG C S, DUAN B Y, ZHANG F S, et al. Coupled structuralelectromagnetic-thermal modelling and analysis of active phased array antennas[J]. IET microwaves, antennas \& propagation, 2010, 4(2): $247-257$.
7. DU Y W. Telegraphy design of radome. Beijing: National Defense Industry Press, 1993.

8. XU W Y, DUAN B Y, LI P, et al. Multiobjective particle swarm optimization of boresight error and transmission loss for airborne radomes[J]. IEEE Transactions on Antennas and Propagation, 2014, 62(11): 5880-5885.

Baoyan DUAN is currently an Academician of Chinese Academy of Engineering and a full Professor of Electromechanical Engineering, Xidian University, China. He received the Ph.D. degree in Mechanical Engineering from Xidian University in 1989. From 1991 to 1994, he studied as Postdoctoral Fellow at Liverpool University. His research interests include mechatronics, electromechanical coupling, structural optimization, and CAD/CAE. E-mail: byduan@xidian.edu.cn

Congsi WANG is currently a Professor at Key Laboratory of Electronic Equipment Structure Design of Ministry of Education, Xidian University, China. He received the B.S., M.S. and Ph.D. degrees in Electromechanical Engineering from Xidian University in 2001, 2004 and 2007, respectively. His research interests are primarily in the area of electromechanical coupling of electronic equipments with emphasis on the modeling, influencing mechanism, design and application of structural-electromagnetic-thermal coupling of antennas including phased array antennas, reflector antennas, deployable antennas and so on. E-mail: congsiwang@163.com

Wei WANG born in 1980, is currently an Associate Professor at Key Laboratory of Electronic Equipment Structure Design of Ministry of Education, Xidian University, China. He received the B.S., M.S. and Ph.D. degrees in Electromechanical Engineering from Xidian University in 2002, 2005 and 2011, respectively. His main research interests include electro-mechanical coupling theory and multidisciplinary optimization in reflector antennas and phased arrays. Tel: +86-29-88203040; E-mail: wwang@xidian.edu.cn 